Addressing the mental health crisis

Jane Creaton (10)

Excellent research depends on a healthy and supportive working environment in which researchers can flourish, yet there is evidence of a mounting mental health crisis among researchers, which may be exacerbated by the COVID-19 pandemic. What can cancer researchers do to promote good mental health and wellbeing within their research communities?

\section{Crisis, what crisis?}

There is evidence of a growing mental health crisis in the research community, particularly among early career researchers (ECRs, including postgraduate and postdoctoral researchers). In a recent survey of doctoral students ${ }^{1}$, completed by over 6,300 respondents from across the sciences, more than a third reported seeking help for anxiety or depression arising from their studies. In another study ${ }^{2}$, more than half of 3,659 PhD students surveyed experienced symptoms of psychological distress, and $32 \%$ were at risk of developing a common psychiatric disorder. In Wellcome's survey ${ }^{3}$ of over 4,000 UK researchers, $34 \%$ of respondents reported seeking professional help for depression or anxiety during their research career, and $19 \%$ said that they wanted to seek help, but had not done so. There is also evidence that the COVID-19 pandemic is having an adverse impact on the mental health and wellbeing of researchers ${ }^{4,5}$. A survey to explore the impact of the pandemic on the research community ${ }^{4}$ found that $80 \%$ of nearly $5,000 \mathrm{UK}$ ECRs were experiencing some level of mental distress, and $75 \%$ showed low levels of mental wellbeing.

The prevalence of mental health issues has profound consequences for the lives of the individuals affected. However, it may also impact on the quality of research outcomes and, in cancer research, this has serious implications for society as a whole. Poor workplace wellbeing may lead to sickness absence and reduced productivity among ECRs working on research projects. In the longer term, the pool of talented researchers available to lead the next generation of cancer research may be reduced. The Wellcome survey found that the most common reasons given by those who had left the research community were a desire for a better work-life balance (37\%) and a negative impact on wellbeing and mental health $(34 \%)^{3}$.

\section{Causes}

There are many factors associated with mental health issues, ranging from an individual's personal and professional circumstances through to wider structural aspects of research environments. A key factor is working relationships. For doctoral students, the supervisory relationship is central to a successful outcome, and inadequate support can be a significant source of stress. Even where the relationship is good, students may be reluctant to talk about mental health issues, in case this might reflect upon their ability to succeed in their research career. For more established researchers, work relationships and the support provided by managers and colleagues are important factors in the amount of stress experienced. The pandemic has placed considerable strain on some of these relationships, with around a third of researchers feeling that they had not been adequately supported during the pandemic ${ }^{5}$.

More than half of the respondents in the Nature ${ }^{1}$ and Wellcome ${ }^{3}$ surveys reported that long working hours and a poor work-life balance were affecting their personal wellbeing. With increased working at home during the pandemic, boundaries between work and home life are difficult to maintain. These pressures are exacerbated by targets relating to publication and external research income generation. Some workplaces create unreasonable expectations regarding working hours, which are particularly problematic for those with disabilities and/or caring responsibilities. Researchers also reported bullying, harassment and discrimination as endemic in some research environments ${ }^{3}$.

Many of the factors impacting on mental health and wellbeing are structural. Precarious employment is a significant issue, particularly for ECRs employed on a series of short-term contracts. PhD students also experience concerns about the availability of research jobs and financial pressures, particularly if self-funded. The pandemic has increased these uncertainties and heightened concerns about future career prospects.

Certain groups of researchers are at higher risk of poor mental health and wellbeing ${ }^{6}$. These include international students, who are often making significant life and cultural changes alongside the transition to postgraduate research study and tend to be isolated from their sources of support. Other students potentially at risk are those working off campus, part time, with caring responsibilities or who have disabilities. The pandemic has also had a disproportionate impact on these 
researchers and on ethnic minorities who are at higher risk from COVID-19 (REF. ${ }^{5}$ ).

\section{Enhancing mental health literacy}

Mental health literacy includes knowledge of mental health issues, attitudes toward mental health and the ability to seek support. Improved mental health literacy enhances the capacity and willingness of people to seek help. It may also ensure that supervisors, employers and colleagues respond appropriately to signs of mental health problems. Increased knowledge about mental health within the researcher community may also reduce any stigma attached to mental illness. Mental health literacy can be improved through providing information in induction sessions, training for students and supervisors, continuing professional development in workplaces and clear signposting to the support available.

While most universities and institutes provide good mental health and wellbeing services, these may not be tailored to the needs of postgraduate researchers. Workplace support is also widely seen as inadequate, with only $44 \%$ in a UK survey agreeing that their workplace offered adequate wellbeing support and only $28 \%$ agreeing that workplace wellbeing initiatives were appropriate to their needs ${ }^{3}$.

\section{Promoting good support networks}

Research has highlighted the importance of good social support networks in reducing the risk of mental health disorders and enhancing recovery if they do develop ${ }^{7}$. Regular contact with supportive peers, supervisors and mentors can combat feelings of loneliness and isolation that may impact negatively on mental health, and so alternative online arrangements are vital during the pandemic. As supervisors and line managers are often best placed to identify signs of stress or other mental illness, universities and employers need to provide adequate support and training in mental health and wellbeing for people in these roles. The research community can ensure that ECRs feel integrated by facilitating membership of formal networks and providing opportunities for connecting with colleagues for social and work related activities. These, together with formal and informal mentoring schemes and peer networks, can contribute to a positive environment in which all researchers can flourish.

\section{Improving research culture}

Aspects of research environments that are detrimental to good mental health and wellbeing include pressures to publish and generate research income, long working hours and a culture of competitive individualism. There is a need to manage expectations around working hours, breaks and holidays and to recognize that increased sickness, high turnover, lower productivity and quality of work are associated with poor mental health. There are actions ${ }^{8}$ that organizations can take to improve research culture. Online lab groups, mentoring and networking programmes can maintain strong support networks within the scientific community ${ }^{9}$. A key factor in successful organizational change is senior researchers leading by example in modelling healthy working practices.

The UK's Concordat to Support the Career Development of Researchers ${ }^{10}$ is an example of a national initiative to improve research culture through agreement between researchers, funders and employers. As a signatory, Cancer Research UK has committed to the key principles, such as promoting good mental health and wellbeing through effective management of workloads and people; implementing policies for tackling discrimination, bullying and harassment; regularly reporting on the quality of the research environment and culture and using the outcomes to improve institutional practices.

\section{A way forward}

Promoting good mental health and wellbeing for researchers requires a range of strategies. Researchers can take some responsibility for their own wellbeing through increasing their mental health literacy, taking up opportunities for engaging with their research community and building good support networks with colleagues and supervisors. Institutions can promote healthy and supportive working environments, set expectations for workloads and work-life balance, and ensure discrimination, bullying and harassment are tackled. However, there are deeper structural factors that contribute to poor mental health and wellbeing that require action at national level if research communities are to recruit and retain the next generation of researchers. The pandemic provides an opportunity to rethink how we can build more supportive and resilient research communities to support those contributing to cancer research.

1. Woolston, C. PhDs: the tortuous truth. Nature 575, 403-406 (2019).

2. Levecque, K., Anseel, F., De Beuckelaer, A., Van der Heyden, J. $£$ Gisle, L. Work organization and mental health problems in PhD students. Res. Policy 46, 868-879 (2017).

3. Moran, H. et al. Understanding Research Culture: What researchers think about the culture they work in. Wellcome Open Res. 5, 201 (2020).

4. Byrom, N. The challenges of lockdown for early-career researchers. elife 9, e59634 (2020).

5. Woolston, C. Pandemic darkens postdoc's work and career hopes. Nature 585, 309-312 (2020)

6. Metcalfe, J., Wilson, S. \& Levecque, K. Exploring wellbeing and mental health and associated support services for postgraduate researchers, https://re ukri.org/documents/2018/mental-healthreport/ (2018)

7. Saltzman, L., Hansel, T. \& Bordnick, P. Loneliness, isolation, and social support factors in post-COVID-19 mental health. Psychol. Trauma 12, 55-57 (2020).

8. Chaplin, K. \& Price, D. 7 ways to promote better research culture https://www.weforum.org/agenda/2018/09/7-ways-to-promotebetter-research-culture/ (2018).

9. Levine, R. \& Rathmell, W. COVID-19 impact on early career investigators: a call for action. Nat. Rev. Cancer 20, 357-358 (2020).

10. Vitae. The Concordat to Support the Career Development of Researchers. https://www.vitae.ac.uk/policy/concordat/full (2019).

\section{Competing interests}

The author declares no competing interests. 